\title{
COMOB: A MATLAB Toolbox for Sensor Placement and Contaminant Event Monitoring in Multi-zone Buildings
}

\author{
Alexis Kyriacou $^{\mathrm{a}, *}$, Michalis P. Michaelides ${ }^{\mathrm{b}}$, Demetrios G. Eliades ${ }^{\mathrm{a}}$, Christos G. Panayiotou ${ }^{\mathrm{a}}$, Marios M. Polycarpou ${ }^{\mathrm{a}}$ \\ ${ }^{a}$ KIOS Research and Innovation Center of Excellence and the Department of Electrical and Computer Engineering, University of Cyprus, CY-1678 Nicosia, Cyprus \\ ${ }^{b}$ Department of Electrical Engineering, Computer Engineering and Informatics, Cyprus University of Technology, \\ 30 Archbishop Kyprianos Str., CY-3036 Lemesos, Cyprus
}

\begin{abstract}
The monitoring of the indoor air quality against the presence of contaminant sources is of paramount importance since it directly influences the occupants comfort, productivity, health and safety. Various approaches exist in the literature for monitoring and controlling the building environment. However, there is an identified lack of automated tools for evaluating, testing and comparing such approaches. This paper presents COMOB (COntaminat MOnitoring in Buildings), a developed MATLAB toolbox for sensor placement and contaminant monitoring in buildings. Specifically, the COMOB toolbox realizes an offline sensor placement procedure for positioning the contaminant sensors inside the building, such that the harmful effects due to a possible airborne contaminant event are minimized. In addition, an online contaminant monitoring approach is also implemented for detecting and isolating contaminant events in the building interior. Besides the aforementioned algorithms, the modularity of the COMOB toolbox facilitates the implementation of various other algorithms related to air-quality monitoring in buildings. In particular, the COMOB toolbox is able to generate multiple airflow and contaminant concentration data-sets by varying the building parameters; thus enabling the comprehensive and systematic evaluation and testing of the developed algorithms. Finally, the functionalities of the COMOB toolbox are demonstrated through a case-study corresponding to a real building model. COMOB is released under an open-source license, and is available at https://github.com/KIOS-Research/COMOB-Toolbox.
\end{abstract}

Keywords: Multi-zone buildings, contaminant event monitoring, multiple scenarios, detection and isolation, sensor placement

\section{Intoduction}

Indoor air quality plays a significant role in today's modern society, since, according to recent studies, people in the United States spend on average $87 \%$ of their time indoors [1]. Occupants' comfort, health, productivity and safety may be compromised due to various airborne contaminants as a result of accidents, faulty equipment or malicious acts. Advancements in sensing technologies have enabled the utilization of sensor networks for monitoring the indoor environmental conditions such as air temperature, humidity and contaminant concentration. Real-time collected data can be used for controlling the environmental conditions while facilitating the accurate and prompt identification of contaminant sources to determine appropriate response solutions such as: (i) isolating contaminated spaces, (ii) initiating evacuation plans and (iii) cleaning contaminated spaces by removing sources, ventilating and filtering air.

For indoor air and contaminant simulation there are two main modeling approaches: Computational Fluid Dynamics (CFD)

${ }^{*}$ Corresponding author. KIOS Research and Innovation Center of Excellence and the Department of Electrical and Computer Engineering, University of Cyprus, CY-1678 Nicosia, Cyprus, Tel: +357 22893450, Fax: +357 22893455

Email address: akyria09@ucy.ac.cy (Alexis Kyriacou) and multi-zone. CFD method can provide a detailed description of the spatial distribution and evolution of air pressure, velocity, temperature, humidity and contaminants but it is also associated with a high computational overhead [2]. On the other hand, multi-zone models are computationally efficient and they represent a building as a network of well-mixed zones. Zones are connected by discrete airflow paths such as doors, windows, wall cracks, ducts and hallways. The most popular multi-zone simulation programs are COMIS [3] by Lawrence Berkeley National Laboratory (LBNL) and CONTAM [4] by the US National Institute of Standards and Technology (NIST).

In the last decade, there have been a number of software and toolboxes proposed in the literature to address various modeling and decision support issues in buildings including a computeraided design for integrated planning and design by optimization (HudCAD) [5], modeling and identification of thermal systems in buildings (LORD) [6], estimation of the thermal properties of building components (IDENT) [7], comparison of building's actual performance and expected performance in real time [8], simulation of buildings energy performance considering probabilistic models of occupants behavior (WinProGen) [9], estimation of life cycle $\mathrm{CO}_{2}$ emissions of buildings in Sri Lanka [10] and value-based decision support for renovation of buildings 
(REDIS) [11]. To the best of our knowledge, there is currently no toolbox dealing with the important issues of contaminant sensor placement or contaminant detection. In particular, there is an identified lack of a toolbox that can provide a common framework for testing and validating methodologies related to the monitoring against contaminants in the indoor building environment.

A plethora of approaches have been proposed for the problems of contaminant sensor placement and contaminant detection, localization and identification. Sensor placement methodologies are either utilizing stationary sensors or mobile sensors, while they can be based on different contaminant dispersion models (e.g., multi-zone and CFD models) depending on their target degree of accuracy and problem size. In [12], a genetic algorithm is utilized to find the sensor placement that minimizes the detection time and occupants exposure for particular contaminant release scenarios, using a mutli-zone model. A probability-based inverse modeling method is presented in [13], where both multi-zone and CFD models are utilized for designing the sensor network, in order to increase the contaminant detection sensitivity. A comparison between different modeling approaches for sensor placement in terms of sensitivity and coverage for contaminant detection can also be found in $[14,15]$. A multi-objective optimization formulation based on a multi-zone model is presented in [16], that finds the locations of sensors that minimize the harmful effects of a set of possible contaminant events on the occupants. More recently, the contaminant dispersion has been characterized by a dynamical system in [17] and, based on its observability gramian matrix, the sensors' locations inside a zone were determined, while aiming to maximize the sensing coverage and minimize the detection time in the entire space, and follow sensor location restrictions. A sensor placement configuration that increases the number of successfully localized sources is found in [18], where a backward advection-diffusion problem is solved to obtain the observable region of each sensor. Non-stationary sensors located on robots were also proposed and tested through simulation for source identification and localization [19, 20, 21]. According to the sensor location and type, various contaminant monitoring and diagnosis methodologies can be applied that differ mainly in terms of the assumed a priori knowledge for the plant, the location, the type and the number of simultaneous sources they can handle. Real time identification of indoor pollutants using neural networks are presented in $[22,23]$ where the number and allocation of the indoor contaminant sensors is also optimized. A multi-source identification approach was presented in [24] that assumed known source location and limited number of possible simultaneous releases. Centralized and distributed state-space methods, based on multi-zone models were presented in [25] and [26] respectively, for detecting and isolating contaminant sources while considering modeling uncertainty and sensor noise. Inverse modeling methods, where the contaminant identification starts at the sensor and trails back to the source location have also been widely used in the literature $[27,28]$. Recent examples include the adjoint probability-based method in [29], assuming unknown pollutant release time and unsteady flows, and the identification of multiple simultaneous temporal [30] and instantaneous [31] pollutant sources. Single and multiple contaminant sources with unknown locations and release rates were also successfully identified in [32, 33] using a variational continuous assimilation method, and in [34] using Gaussian process-based emulation to perform inference on the location of the contaminant sources.

The aforementioned literature presents an overview of recent approaches that have been proposed for contaminant sensor placement and monitoring of the IAQ. The contaminant sensor placement solutions vary in terms of their objectives (i.e., maximize sensing coverage, minimize detection delay), while only a few works account for the building utilization and people distribution (i.e., zone occupancy). The contaminant monitoring approaches use either model-based methodologies, that assume the presence of a physical model for performing the estimation task, or data-driven approaches, that utilize learning to either construct a model (from the data) or a scenario database before the event. Differences are also observed in terms of the assumed architecture, centralized or distributed, the consideration of modeling uncertainty, the use of a priori information for solving the problem (i.e., the building flows or the release time and possible locations for the contaminant source) and the way validation is performed (number of case studies). In general, there is a large gap identified between modeling software and decision approaches, mainly due to the absence of a single unifying framework that enables their interoperability and coordination. Validating and comparing these approaches requires a large number of generated scenarios and unifying structures of models and data, which are usually non-existent. Such obstacles are the main reasons for the limited availability of reproducible research outputs and in turn, the limited number of monitoring and decision support tools for the indoor building environment.

This work is focused on describing the development and the capabilities of COMOB (COntaminant MOnitoring in Buildings), a MATLAB toolbox for sensor placement and contaminant monitoring in buildings. The contributions of the COMOB toolbox are threefold. First, the realization of an offline methodology for determining the contaminant sensor locations inside the building in order to minimize the negative effects to the occupants following a possible contaminant event and taking into consideration the building's utilization. Secondly, the realization of an online model-based procedure for contaminant detection and isolation (CDI), that utilizes both centralized and distributed fault ${ }^{1}$ diagnosis tools for determining the presence and the location of the contaminant source(s) in the building interior under conditions of noise and modeling uncertainty. Lastly, the seamless generation of benchmark datasets related to the presence of contaminants in multi-zone buildings for testing and validating the developed methodologies for air-quality monitoring in buildings. In fact, the generated datasets combined with the modular architecture of the COMOB toolbox enable the development and evaluation of various methodologies (such as evolutionary computation methods, optimization pro-

${ }^{1}$ Note that under the state-space representation, the contaminant release is modeled as a fault in the process which we aim to detect and isolate. 
blems and fault diagnosis algorithms [35, 36, 37, 38]) in buildings' related research. This is particularly challenging since it is rather difficult to define and propose benchmarks and/or testbeds for testing and validating the developed methodologies in dynamic non-stationary environments.

In this work, we present a case study based on a 14-zone model of a real building referred to as the "Holmes House" ${ }^{2}$, in order to demonstrate the use and the different functionalities of the COMOB toolbox. Given the building model, and after specifying acceptable values and ranges for various parameters associated with the environment (temperature, wind speed and direction), the contaminant source (location, duration and onset time) and the building (opening status of doors and windows); the COMOB toolbox can automatically generate a number of different datasets corresponding to the specified scenarios. Based on the generated scenarios, COMOB can then determine the optimal locations for different numbers of available contaminant sensors. Furthermore, assuming a particular sensor placement, the COMOB toolbox can detect the existence of a contaminant source and identify its location inside the building. We should point out that the presented algorithms and case study are only a small sample of the COMOB toolbox's capabilities.

The rest of the paper is organized as follows: In Section 2, the theoretical background for the sensor placement and contaminant detection and isolation algorithms is provided. Next, the software architecture of the COMOB toolbox is described in Section 3. The sensor placement case study followed by the contaminant detection and isolation case study, both using the Holmes building model, are presented in Section 4. Finally, Section 5 concludes the paper and provides information about software updates planned in future releases.

\section{Theoretical Background}

\subsection{State-space Method for Multi-zone Buildings}

Both the indoor contaminant dispersion dynamics and the algorithms utilized in this paper, are based on a state-space formulation for multi-zone buildings that was first developed in 2012 [40] to address the problem of contaminant event monitoring and control in indoor building environments. Let $\mathbb{R}$ represent the set of real numbers and $\mathbb{B}=\{1,0\}$ the set of binary. According to the proposed formulation, the state space equations for contaminant dispersion in an indoor building environment with $n$ zones can be presented in the following general form,

$$
\begin{aligned}
& \dot{x}(t)=A x(t)+Q^{-1} B u(t)+h(x(t), u(t))+Q^{-1} D d(t)+Q^{-1} G g(t) \\
& y(t)=C x(t)+w(t),
\end{aligned}
$$

where, $x \in \mathbb{R}^{n}$ represents the concentrations of the contaminant in the building zones, $A \in \mathbb{R}^{n \times n}$ is the state matrix and $B \in \mathbb{B}^{n \times p}$ is a zone index matrix for the controllable inputs (e.g., doors, windows, fans and air handling units) represented

${ }^{2}$ Refers to a low-rise residential house model used by J.D. Holmes and then scaled up for Simulating Airflow and Contaminant Transport in and around Buildings [39]. by $u \in \mathbb{R}^{p}$. The vector field $h: \mathbb{R}^{n} \times \mathbb{R}^{p} \mapsto \mathbb{R}^{n}$ characterizes the modeling uncertainties, $D \in \mathbb{B}^{n \times q}$ is a zone index matrix for the disturbances represented by $d \in \mathbb{R}^{q}$, and $G \in \mathbb{B}^{n \times s}$ is a zone index matrix for the generation sources represented by $g \in \mathbb{R}^{s}$, while $Q \in \mathbb{R}^{n \times n}$ is a diagonal matrix with the volumes of the zones (i.e. $Q=\operatorname{diag}\left(q_{1}, q_{2}, \ldots, q_{n}\right)$ where $q_{i}$ is the volume of zone $i$ ). In the second equation, $y \in \mathbb{R}^{m}$ represents the sensor measurements, $C \in \mathbb{B}^{m \times n}$ is a zone index matrix for the sensor locations and $w \in \mathbb{R}^{m}$ stands for additive measurement noise. Additional details in relation to the state-space formulation for multi-zone buildings can be found in [40]. Note that a similar state-space formulation has been commonly used by the fault diagnosis community to represent models of uncertain non-linear systems [41]. In the context of fault diagnosis, the final term in (1), i.e. $Q^{-1} G g(t)$, is used to describe process faults that disturb the normal system operation.

\subsection{Sensor Placement}

The sensor placement methodology, as utilized in the software, is described in this section. A detailed description is presented in [16]. The goal is to solve a combinatorial problem for deciding where to install contaminant sensors inside a building, in order to reduce the possibility of severe damage due to an airborne contaminant event which may affect the building operation and the health of the occupants.

In the following, the formulation is based on the multi-zone model described in the previous section. The state-space equations for contaminant dispersion in an indoor building environment with $n$ zones are described by:

$$
\dot{x}=A\left(p_{x}\right) x+\phi\left(p_{x}, p_{\phi}\right) .
$$

Note that compared to the first equation of the general formulation in (1), we do not consider here controllable inputs, disturbances or modeling uncertainty, while the time dependency has been removed to simplify the notation. The vector $x \in \mathbb{R}^{n}$ as before, represents the concentration of the contaminant in the building zones (measured in mass per volume). The state matrix $A \in \mathbb{R}^{n \times n}$ models the changes in the contaminant concentrations between the different building zones as a result of the airflows. Compared to (1), the state matrix is now parametrized by the vector $p_{x}$, which is comprised of the wind-direction, the wind-speed, the ambient temperature, the volumes of all zones, the temperatures in all zones and the opening status of all doors, windows and fans. The term $\phi \in \mathbb{R}^{n}$ represents the contaminant mass release into the building due to an accident or an attack, and $p_{\phi}$ is a vector comprised of the locations where contaminants are released, their release duration, as well as their release rates.

For specific contaminant sources, environmental and building parameters, an impact value, indicated by the variable $z_{k}$, is defined in [16] to model the damage caused to the occupants during a contamination event in the $k$-th zone and its dynamics are described by:

$$
\dot{z}_{k}=f_{z}\left(x_{k} ; p_{z}\right)
$$


where $f_{z}(\cdot)$ is a function of the contaminant concentration $x_{k}$ and the set of parameters $p_{z}$ which include the average daily zone occupancy (proportional to the actual time that the zone is occupied), the inhalation rate (which depends on the building/zone functionality), and the concentration threshold of the sensors. Note that the impact value can also be influenced by other factors, such as the total mass inhaled and the number of affected occupants or zones.

To illustrate the construction of the impact value for a specific contamination and airflow scenario, consider the use of the "contaminant mass inhaled" as our impact metric and let $h_{k}$ be the daily average rate of air volume inhaled by all occupants within the $k$-th zone. The inhaled air volume can in turn depend on the age group, sex and the activity intensity of each occupant [42]. Hence, the impact state dynamics for the $k$-th zone can then be expressed as:

$$
\dot{z}_{k}(t)=x_{k}(t) h_{k}
$$

In addition, consider that $T_{d_{k}}$ represents the time at which the sensor in the $k$-th zone becomes alarmed based on a predefined threshold (i.e., the minimum concentration based on the manufacturer specifications). The sensor threshold signifies the minimum contaminant concentration that is considered harmful for the occupants. Therefore, the solution of (4) at time $T_{d_{k}}$ represents the impact of the inhaled contaminant on the occupants and is given by:

$$
z_{k}\left(T_{d_{k}}\right)=z_{k}(0)+h_{k} \int_{0}^{T_{d_{k}}} x_{k}(t) d t .
$$

Multiple contaminant release scenarios are considered and used to construct a finite set $\mathcal{P}$ which is comprised of $N_{p}$ elements corresponding to all the combinations of various contaminant sources, environmental and building parameters. For each contaminant scenario in $\mathcal{P}$, the indoor contaminant dispersion dynamics are simulated for a period of $T$ hours. Let $\Omega$ be the overall-impact matrix with respect to the impact value $z_{k}$ as:

$$
\Omega=\left[\begin{array}{ccc}
\Omega_{1,1} & \cdots & \Omega_{1, n} \\
\vdots & \ddots & \vdots \\
\Omega_{N_{p}, 1} & \cdots & \Omega_{N_{p}, n}
\end{array}\right],
$$

such that $\Omega_{i, k}=f_{\omega}\left(z_{k}\right)$ represents the impact risk generated due to the $i$-th contamination scenario from $\mathcal{P}$, assuming a sensor is monitoring the $k$-th zone.

Finally, the contaminant sensor placement problem in buildings is formulated as a multi-objective optimization problem by considering the number of installed sensors, the average impact risk and the worst case impact risk. The Pareto Front solutions are computed using either an exhaustive search (for small problems) or a multi-objective evolutionary optimization approach.

\subsection{Contaminant Event Detection and Isolation}

Current contaminant sensors become alarmed based on constant pre-determined thresholds. However, there is a tradeoff between their high sensitivity to contaminant concentration changes and the frequency of false alarms. In addition, while a contaminant may be detected, little or no information is given for its location in the building and its characteristics (e.g., contaminant release rate). The presented CDI approach utilizes model-based estimation of the contaminant concentration and adaptive thresholds that consider measurement noise and modeling uncertainty; thus increasing detection sensitivity, eliminating false alarms and providing the ability of isolating the location of the contaminant source.

In this section, we provide an overview of the theoretical results used in the development of the "Contamination Detection and Isolation Module". The COMOB toolbox includes the implementation of both centralized and distributed CDI algorithms that are described in detail in [25] and [26] respectively. Additionally, using the multi-zone modeling methodology presented in Section 2.1, the state-space equations for contaminant dispersion in an indoor building environment with $n$ zones are presented for both the centralized and distributed CDI algorithms.

\subsubsection{Centralized State-Space Formulation}

Given an $n$-zone building, the contaminant dispersion in the indoor environment can be described by the following multizone model:

$$
\begin{aligned}
\Sigma: \quad \dot{x}(t) & =(A+\Delta A) x(t)+Q^{-1} B u(t)+Q^{-1} G g(t), \\
y(t) & =C x(t)+w(t),
\end{aligned}
$$

Note that compared to (1), disturbances are not considered, while the modeling uncertainties (resulting from changing wind speed, wind direction and leakage openings) in the building envelope are collectively accounted by the term $\Delta A \in \mathbb{R}^{n \times n}$. More details on the state-space model formulation can be found in [25].

\subsubsection{Distributed State-Space Formulation}

The overall system $\Sigma$ is partitioned into $K$ interconnected subsystems $\Sigma_{I}, I \in\{1, \ldots, K\}$, where each subsystem corresponds to the dispersion of the contaminant in an area of $n_{I}$ zones, described by:

$$
\begin{aligned}
\Sigma_{I}: \quad \dot{x}_{I}(t)= & \left(A_{I}+\Delta A_{I}\right) x_{I}(t)+Q_{I}^{-1} B_{I} u_{I}(t)+ \\
& +Q_{I}^{-1} G_{I} g_{I}(t)+\left(H_{I}+\Delta H_{I}\right) \zeta_{I}(t), \\
y_{I}(t)= & C_{I} x_{I}(t)+w_{I}(t),
\end{aligned}
$$

where $x_{I} \in \mathbb{R}^{n_{I}}, u_{I} \in \mathbb{R}^{p_{I}}$ and $y_{I} \in \mathbb{R}^{m_{I}}$ are the local state, local control input in the form of doors, windows, fans and air handling units, and local measured output vectors, respectively, and $\zeta_{I} \in \mathbb{R}^{\ell_{I}}$ is the vector of the interconnection variables. The term $g_{I} \in \mathbb{R}$ represents the local contaminant source and $w_{I} \in \mathbb{R}^{m_{I}}$ corresponds to the local noise vector. Note that, in the distributed formulation, we assume a maximum of a single contaminant source per subsystem. Finally, $A_{I}, \Delta A_{I}, H_{I}, \Delta H_{I}, Q_{I}, G_{I}$ and $C_{I}$ are sub-matrices of the centralized system $\Sigma$ of appropriate dimensions. 


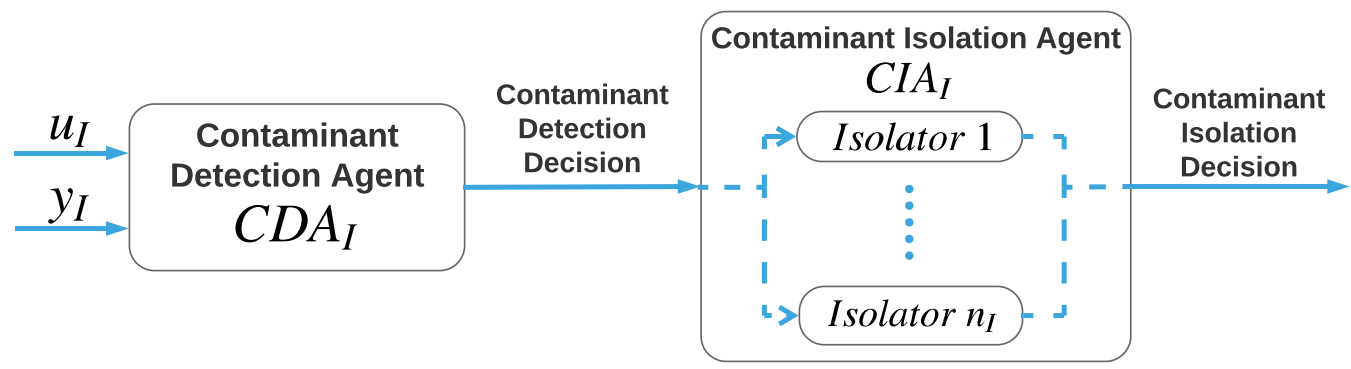

Figure 1: Architecture of detection and isolation of a single contaminant source.

\subsubsection{Contaminant Detection and Isolation}

The contaminant source in the centralized and distributed contaminant dispersion models described in (7) and (9) respectively, is modeled as a fault in the process. Therefore, advanced fault diagnosis tools ([43, 44, 45, 46, 47]) can be used to detect and isolate the contaminant source(s). The remainder of the paper will focus on the implementation and demonstration of the distributed CDI methodology since the centralized case is perfectly analogous to the distributed case with only one subsystem. In general, distributed CDI has significant benefits over the centralized approach due to (i) the lower complexity in designing the CDI method for fewer zones, (ii) the improved scalability from the easy incorporation of additional zones, (iii) the increased reliability due to the lack of a single point of failure, (iv) the reduction of communication requirements since there is no need of transmitting all the information globally, and (v) the improved ability in handling multiple contaminant sources.

For the distributed case, the building is considered as a collection of interconnected subsystems where a contaminant detection agent (CDA) and a contaminant isolation agent (CIA) are designed for each subsystem. Agents are responsible for detecting and isolating a contaminant source while they are allowed to exchange measurement information with their neighboring agents. The general architecture of the algorithms for detecting and isolating a single contaminant source appears in Fig. 1. The Contaminant Detection Agent, $C D A_{I}$, $I \in[1, \ldots, K]$ is responsible for estimating the contaminant concentration in subsystem $\Sigma_{I}$ under the "no source" hypothesis. It then compares the contaminant concentration as measured by the sensors to the estimated one. A contaminant is then detected if the difference (residual) between the actual and the estimated concentration of at least one sensor exceeds the adaptive threshold. The adaptive thresholds are designed as to bound the residual under healthy conditions where no contaminant source is present. Following the contaminant detection, the Contaminant Isolation Agent, $C I A_{I}$, activates a bank of $n_{I}$ isolators, one for each building zone that the $C D A_{I}$ is monitoring. Each $k$-th, $k \in\left[1, \ldots, n_{I}\right]$ isolator estimates the contaminant concentration inside the $n_{I}$ building zones under the "source present in $k$-th zone" hypothesis. Then it compares the actual contaminant concentrations as measured by the sensors to the estimated ones. If the residuals produced by the $k$-th isolator surpass the threshold in any zone, then the $k$-th zone is excluded from the possible candidate zones for the source location. In order for the contaminant source to be successfully isolated, all but one zones need to be excluded.

Further details on the algorithms including the derivation of the residuals and the adaptive thresholds can be found in [25] and [26] for the centralized and distributed CDI respectively.

\section{COMOB Toolbox Description}

\subsection{Revisions}

The first COMOB Toolbox versions were published in 2013 as separate MATLAB toolboxes for CDI and Sensor Placement (see [48] and [49] respectively). The software revision presented in this paper, combines the methodologies into a single unified software, while it also further expands their capabilities. Firstly, it upgrades the software to include the distributed statespace model for contaminant dispersion and by extension the distributed CDI architecture including the selection of subsystem configuration and the computation of each subsystem's modeling and interconnection uncertainty. Secondly, it provides an interactive visualization of the sensor placement results in the main building diagram and an intuitive interface for the visualization of the generated scenarios and CDI results. In addition, among the new important features lies the ability of exporting the results into MATLAB table data structures for easier accessing, utilisation and transferring between applications. Finally, the graphical user interface of the software has been revamped to incorporate the new functionalities while retaining its user friendly character.

\subsection{COMOB Toolbox Architecture}

The COMOB Toolbox Architecture is depicted in Fig. 2. The software acquires the building information from CONTAM project files, which are comprised of details regarding walls, floors, ceilings, air-handling systems, ducts, weather conditions etc. The "Read Project File" module opens CONTAM project files (i.e., *.prj), extracts the information which are related to the building parameters and stores them in a MATLAB structure "B". In addition, the building zone schematics are extracted and this information is used by the GUI of the toolbox to plot the building schematics. 


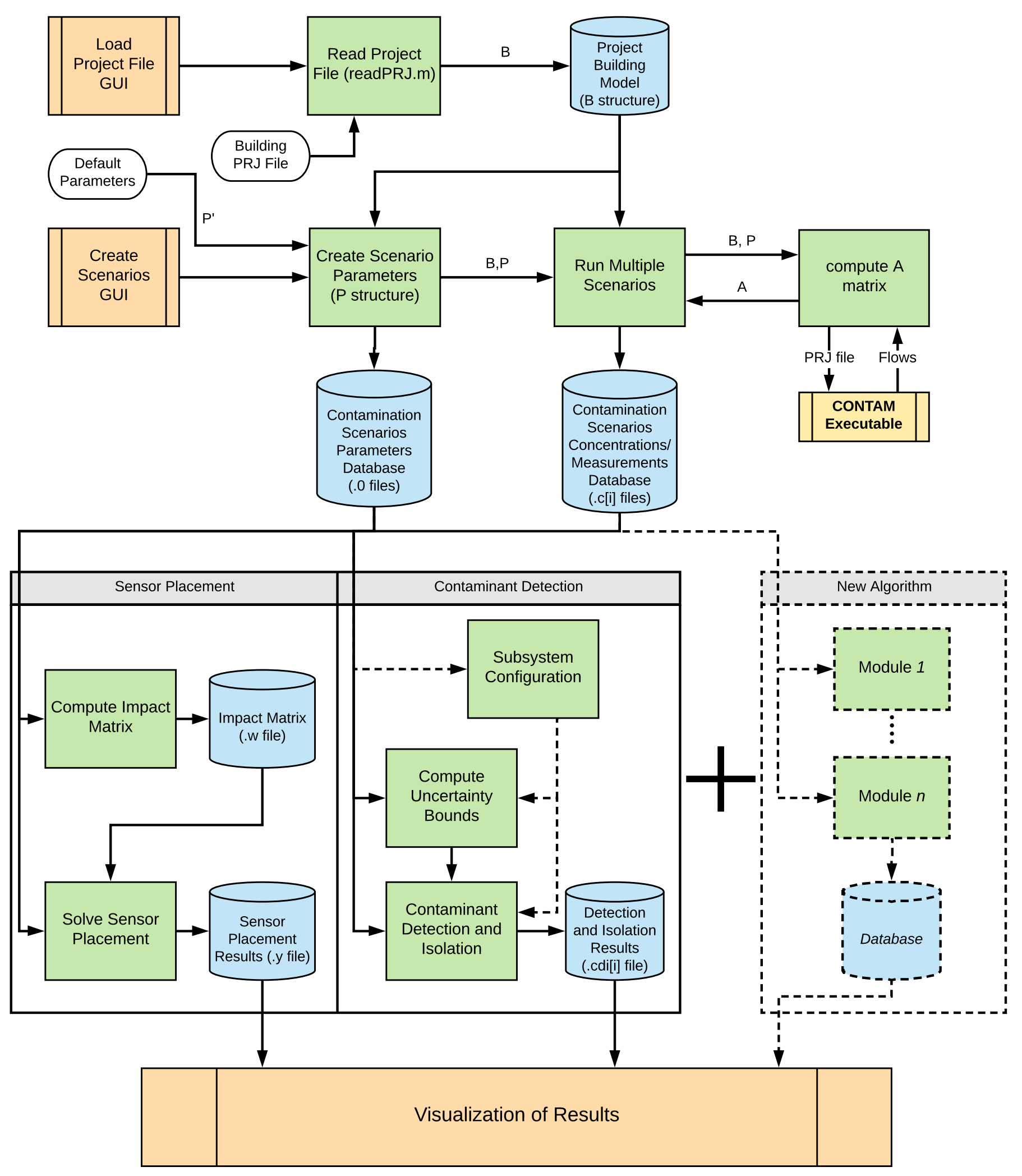

Figure 2: The software architecture of the COMOB toolbox. 
The information from structure " $\mathrm{B}$ " is then used by the "Create Scenario Parameters" module. This module assists in specifying, through the GUI, the building parameters for constructing a single or multiple simulation scenarios. The different contamination scenario parameters, along with structural and environmental information are stored in the Contamination Scenarios File (i.e., *.0) and in a structure "P".

The data stored in the Scenarios File is used by the "Run Multiple Scenarios" module, which iteratively through the "Compute A Matrix" module, communicates with the CONTAM engine to compute the air-flows (i.e., matrix A) in the building interior for different environmental conditions. Note that the "Compute A Matrix" uses structures "B" and "P" to automatically configure a CONTAM project files (i.e., *.prj) and call the CONTAM engine. When the air-flows have been computed, the module simulates using the contaminant dispersion model in (7) the different contaminant release scenarios and computes the contaminant concentration at each building zone. The contaminant concentrations are then stored in one or more Concentration Files (i.e., *.c1, *.c2, ...).

The Sensor Placement algorithm consists of two modules. The first module, "Compute Impact Matrix", utilizes the data from the contaminant scenarios and simulation databases, as well as data from the GUI (such as the average daily zone occupancy), to calculate the damage caused by some contaminant event, until the time the contaminant exceeds a concentration threshold at a certain zone. The structure generated for all the possible scenarios and all the possible zones is stored in the "Impact Matrix File" (*.w). The second module "Solve Sensor Placement" is used to compute the final solutions, based on the computed impact matrices and the scenarios files. Through the GUI, the user specifies which method to use to solve the problem. For instance, exhaustive search methods would compute all the possible solution combinations, and calculate the Pareto Solutions. The solutions can be visualized graphically on the GUI, and are stored in a Solutions File (i.e., *.y0 for the exhaustive search method and *.y1 for the evolutionary computation method).

Finally, the Contaminant Detection procedure is also formed by two modules. Module "Compute Uncertainty Bounds" is used for calculating the uncertainty bounds for the CDI algorithms. The "Contaminant Detection and Isolation" module, is comprised of state-of-the-art centralized and distributed algorithms for detecting whether a contamination event has occurred in the building, and isolating its zone. The module uses information from the Concentration Files, the Scenarios Files and the uncertainty bounds as computed by the "Compute Uncertainty Bounds" module. All the results produced by the "Contaminant Detection and Isolation" are stored in one or more Detection and Isolation Result Files (*.cdi1,*.cdi2,...). Note that, for the execution of the distributed CDI procedure, a "Subsystem Configuration" module precedes the "Compute Uncertainty Bounds" where the user can either manually define the zones of each subsystem or select one from all possible subsystem configurations.

It is important to mention that, for all the aforementioned modules, intuitive user-friendly interfaces were designed in the proposed software. Also, the COMOB architecture features a completely modular design in that new functionalities and algorithms can be easily integrated into the toolbox in the future as shown in Fig. 2. The main goal of the COMOB toolbox is to facilitate the simulation of multiple contaminant events under varying conditions as well as to store computed results in structured datasets so that they can be easily reused for further analysis. Towards this end, each interface offers capabilities for visualizing and exporting the results in table structures. MATLAB table structures, that store column-oriented data and allow their easy handling and extraction from MATLAB for further processing, have been chosen as the form of exporting the scenario details and results of the provided sensor placement and CDI algorithms. An extra feature of MATLAB tables worth mentioning is that they can be easily exported into JSONformatted text using the " $\mathrm{j}$ sonencode" MATLAB function for further simplification and increased compatibility with possible data transmission requirements.

\section{Case Study}

In this section, different functionalities of the developed COMOB toolbox are demonstrated through a case study corresponding to the "Holmes house" experiment [39].

\section{1. "Holmes house" project}

The main interface of the COMOB toolbox is depicted in Fig. 3, loaded with the project file corresponding to the "Holmes house". As shown in the figure, the building is comprised of 14 zones: a garage (Z1), a storage room (Z2), a utility room (Z3), a living room (Z4), a kitchen (Z5), two bathrooms (Z6 and Z13), a corridor (Z8), three bedrooms (Z7, Z9 and Z14) and three closets (Z10, Z11 and Z12). There are a total of 30 leakage path openings corresponding to windows and doors (P1-P30). It is assumed that natural ventilation is the dominant cause of air movement in the building with wind coming from the east $\left(90^{\circ}\right)$ at a speed of $10 \mathrm{~m} / \mathrm{s}$.

\subsection{Main COMOB toolbox interface}

The COMOB toolbox GUI is divided into 3 main sections as shown in Fig. 3, corresponding to the menu bar on the top, the display area in the middle and some figure control options on the bottom layer. From the File drop-down menu, the user can (i) open a CONTAM project file of a building model, (ii) create and simulate scenarios corresponding to changing airflow and contaminant source properties and (iii) display or modify the building properties of the currently active CONTAM project file. The Algorithms drop-down menu includes options for (i) solving the Sensor Placement problem, (ii) configuring the subsystems for the Distributed Contaminant Detection and Isolation algorithms and (iii) configuring and simulating either the centralized or the distributed contaminant monitoring approaches. Lastly, the Results drop-down menu provides selections for the (i) visualization and extraction of air-flow and contaminant release scenarios results stored in files (*.0 and *.c1,*.c2...), (ii) interactive visualization of sensor 


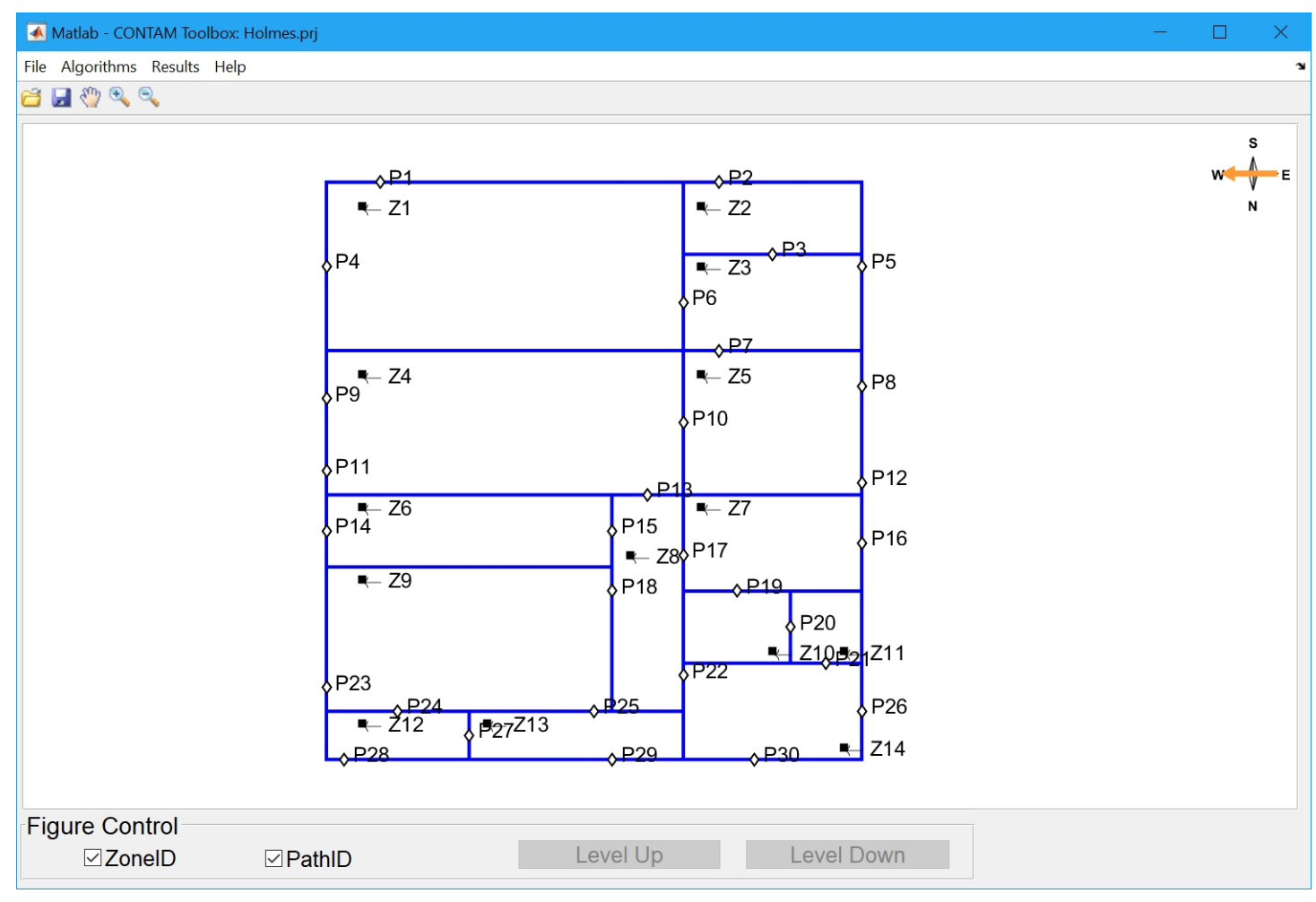

Figure 3: The COMOB toolbox displaying the "Holmes house" project.

placement results in the main GUI and extraction in files (*.w, *.y0 and *.y1) and (iii) visualization and extraction of CDI results in appropriate file formats (*.cdi1,*.cdi $2, \ldots)$.

For the rest of this section, each algorithm will be followed by a demonstration of the corresponding results, if applicable.

\subsection{Create Scenarios}

The interface allows the configuration of scenarios using multiple air-flow and contaminant source parameters and the simulation of the corresponding scenarios. The interface of the Create Scenarios is shown in Fig. 4 corresponding to the case study described in Section 4.1.

In the Scenario Selection sub-section, the generation of either a single or multiple scenarios can be selected. The simulation time and time-step are defined through sub-section Simulation Parameters. Furthermore, the user can choose between configuring the parameters for each scenario (grid method) or randomly generating scenarios (random method). Sub-section Contaminant Source Parameters is used to configure the contaminant source characteristics (i.e., source generation rate, start time and duration) for numerically solving the differential equations (1) and (9), while ambient parameters (i.e., wind direction, wind speed and temperature) can be set in the Environmental Parameters sub-section. In addition, the number and possible source zones of contaminants can be selected through the Number of sources sub-section, while the sensors number and zones can be chosen through the button Select Sensor Zones. The parameters for the path openings and the zones can be selected in a similar way in the Building Parameters sub-section. Note that for all the aforementioned parameters, an option is available for selecting the bounds of deviation from the nominal values as a percentage as well as the number of samples inside those bounds. In the illustrated example, the source generation rate is $500(\mathrm{~g} / \mathrm{h})$ with a $5 \%$ deviation and 2 samples generating 2 different contaminant generation rate scenarios of $[450,550] \mathrm{g} / \mathrm{h}$. Once the parameters have been appropriately selected, they can be saved, producing the corresponding "*. 0 " file and then simulated (i.e., Run button), generating the "*.c1,*.c2,..." files. The simulation of the scenarios involves calling the CONTAM engine for the airflow calculation and numerically solving the differential equations describing the contaminant dispersion in the zones. Previously saved configurations can also be loaded and simulated.

\subsubsection{Scenario Results}

The Scenario Results interface illustrated in Fig. 5 is used for the visualization and extraction in structured datasets of the created scenarios. All the information about the contaminant source characteristics and parameters related to each scenario are presented in a matrix form. Moreover, for each scenario, "*.c[i]", the time series of the concentrations in the different building zones as well as the airflows can be plotted through the Display Scenario Results section. Finally, the information for a single scenario can be exported in a MATLAB table structure. Note that only information for one scenario (i.e., the selected scenario to be illustrated in the table under Scenario Parameters section) can be exported at a single time. Since this toolbox can be used for data analysis studies where generating a large num- 


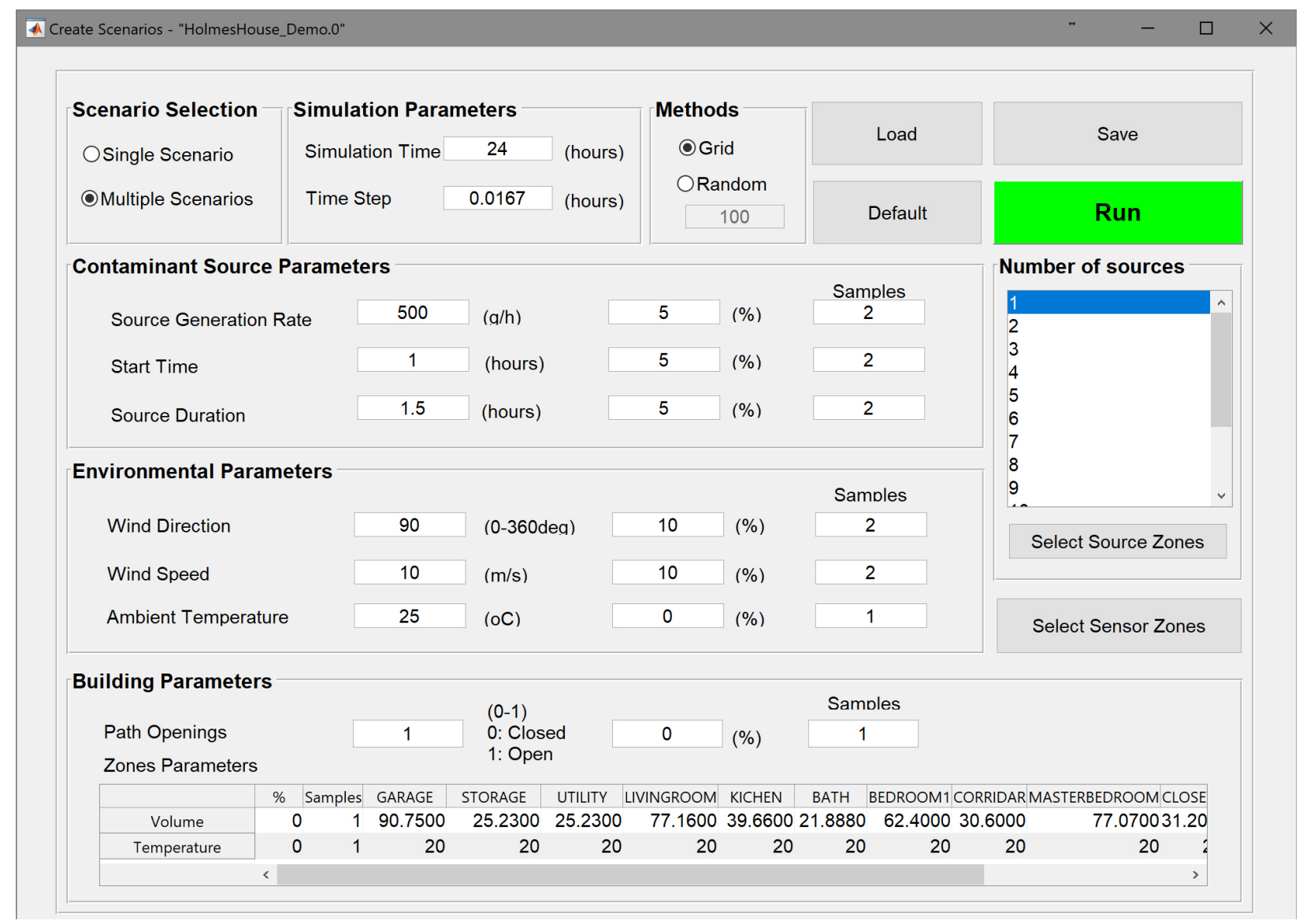

Figure 4: The interface for selecting scenario parameters and constructing the scenarios set.

ber of airflow and contamination scenarios is needed, exporting all generated data into the MATLAB workspace can be limited by software/hardware resources. This can be also witnessed in the illustrated scenario results, where using only 2 samples for each of the contaminant generation rate, the start time and the source duration, a total of $2 \times 2 \times 2=8$ different contamination scenarios for each zone are produced.

\subsection{Sensor Placement}

Using the computed contaminant concentration time-series for each zone, the impact matrix $\Omega$ can be constructed for solving the sensor placement optimization problem. The $(i, j)$-th element of the impact matrix $\Omega$ corresponds to the damage caused due to the $i$-th contamination scenario with respect to having a sensor installed in the $j$-th zone. In this work, the "contaminant mass inhaled" is considered as a metric of the impact damage (3), however different metrics could be implemented as well. The interface for constructing the impact matrix is show in Fig. 6. For each zone, the user can specify an occupancy value, which corresponds to the average number of people residing within a certain zone within a day. For instance, if 10 people reside within a zone for 6 hours each day, the average daily occupancy of that zone would be $10 \times(6 / 24)=2.5$. The inhalation rate depends on the type of work which can be estimated using guidelines [42]. The sensor threshold corresponds to the minimum concentration that a certain sensor becomes alarmed, according to its specifications.

The final step is to solve the optimization problem for sensor placement, using the interface depicted in Fig. 6. The user can select between two methods for constructing the solutions to evaluate the Pareto Front, using the MATLAB's multi-objective evolutionary-based algorithm (gamultiobj) which is suitable for larger problems, and an exhaustive method which is suitable for smaller problems. The user can select different numbers of sensors to solve the optimization. The Pareto Solutions are computed for each different number of sensors.

\subsubsection{Sensor Placement Results}

Following the completion of the Scenario Simulations, and the Sensor Placement algorithm the user can view and export the sensor placement results through the Sensor Placement Results interface, as shown in Fig. 7. The results are plotted in a matrix form, where for each sensor placement, the sensor number, the mean and maximum objective and zones to place sensors are illustrated. Furthermore, the Sensor Placement interface can interact with the main GUI interface in order to visualize the different sensor placement solutions resulting from the algorithm. In particular, the user can select and view in the building schematic (i.e., main GUI interface) any sensor placement solution. For example, Fig. 7 demonstrates the sensor 


\section{Scenario Parameters}

Load Scenarios
HolmesHouse Demo.0

HolmesHouse_Demo.c1 HolmesHouse_Demo.c2 HolmesHouse_Demo.c3 HolmesHouse_Demo.c4 Wind Direction (degrees) WindSpeed (m/..... Source Location Source Generation Rate $(\mathrm{g} / \mathrm{hr})$ Release Time (hr) Duration (hr

\begin{tabular}{|l|l|}
\hline Sc. 1 & 81 \\
\hline Sc. 2 & 81 \\
\hline Sc. 3 & 81 \\
\hline Sc. 4 & 81 \\
\hline Sc. 5 & 81 \\
\hline Sc. 6 & 81 \\
\hline Sc. 7 & 81 \\
\hline Sc. 8 & 81 \\
\hline Sc. 9 & 81 \\
\hline Sc. 10 & 81 \\
\hline Sc. 11 & 81 \\
\hline Sc. 12 & 81 \\
\hline Sc. 13 & 81 \\
\hline Sc. 14 & 81 \\
\hline Sc. 15 & 81 \\
\hline Sc. 16 & 81 \\
\hline Sc. 17 & 81 \\
\hline Sc. 18 & 81 \\
\hline Sc. 19 & 81 \\
\hline
\end{tabular}

$\begin{array}{lllll}9 & . Z 1 & 475 & 0.95 & 1.425 \\ 9 & . Z 1 & 525 & 0.95 & 1.425 \\ 9 & . Z 1 & 475 & 1.05 & 1.425 \\ 9 & . Z 1 & 525 & 1.05 & 1.425 \\ 9 & . Z 1 & 475 & 0.95 & 1.575 \\ 9 & . Z 1 & 525 & 0.95 & 1.575 \\ 9 & . Z 1 & 475 & 1.05 & 1.575 \\ 9 & . Z 1 & 525 & 1.05 & 1.575 \\ 9 & . Z 2 & 475 & 0.95 & 1.425 \\ 9 & . Z 2 & 525 & 0.95 & 1.425 \\ 9 & . Z 2 & 475 & 1.05 & 1.425 \\ 9 & . Z 2 & 525 & 1.05 & 1.425 \\ 9 & . Z 2 & 475 & 0.95 & 1.575 \\ 9 & . Z 2 & 525 & 0.95 & 1.575 \\ 9 & . Z 2 & 475 & 1.05 & 1.575 \\ 9 & . Z 2 & 525 & 1.05 & 1.575 \\ 9 & . Z 3 & 475 & 0.95 & 1.425 \\ 9 & . Z 3 & 525 & 0.95 & 1.425 \\ 9 & . Z 3 & 475 & 1.05 & 1.425\end{array}$

\section{Display Scenario Results}

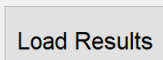

HolmesHouse_Demo.c1

No of Scenario:

1

Concentration Results

Select Zones

$\square$ Plot on the same axes $\square$ Grid on

Plot Concentrations

Airflow Results

\section{Display Airflows}

Display Airflows Graph

Figure 5: The interface for visualization and exporting of the scenario results.

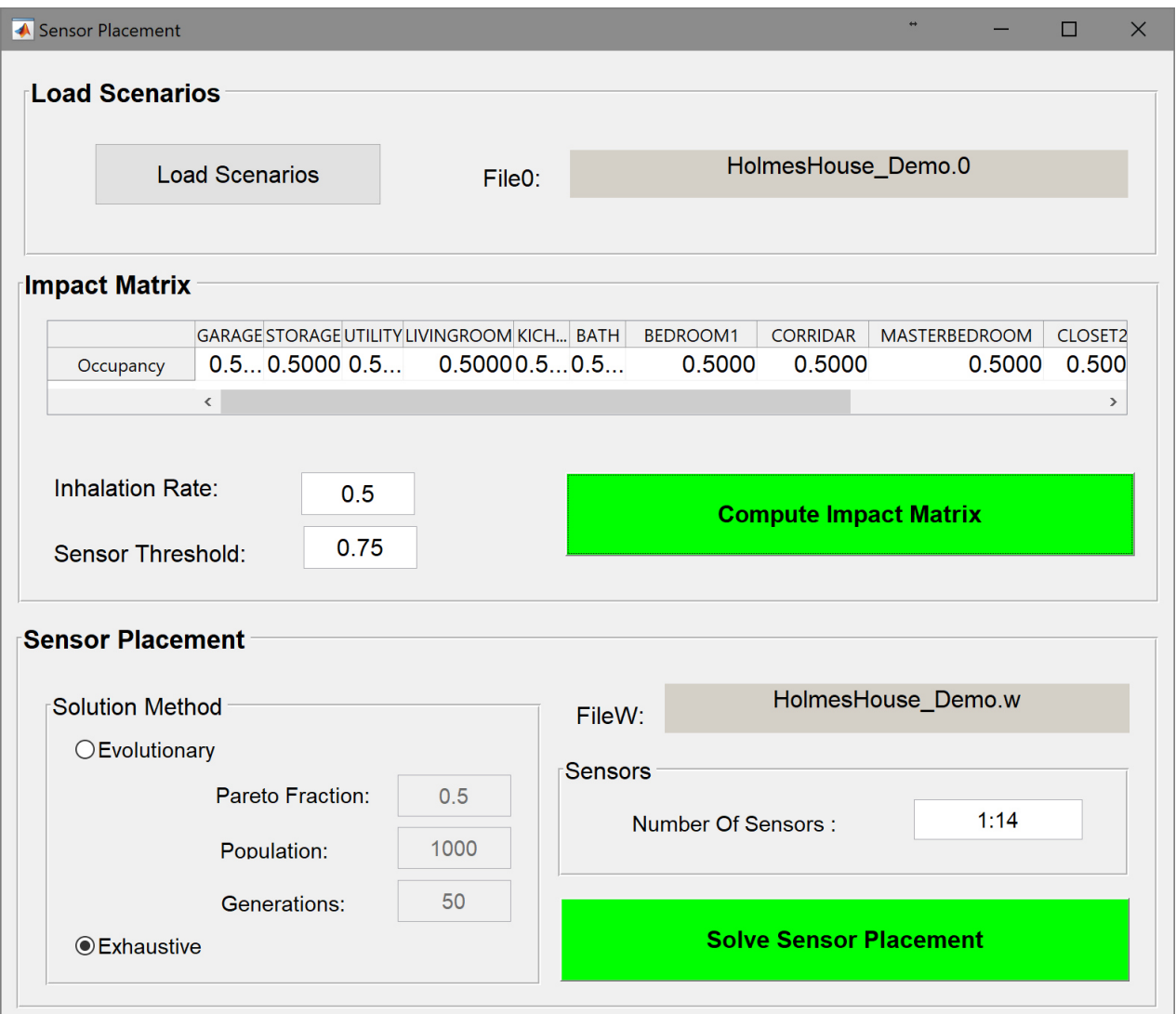

Figure 6: The interface for constructing the impact matrix and solving sensor placement. 
placement 14 , that corresponds to the solution that minimizes the mean and maximum impact risk values using 11 sensors in zones [Z1, Z2, Z4, Z6, Z8, Z9, Z10, Z11, Z12, Z13, Z14]. Note that for the selected sensor placement solution, the shaded zones on the building schematic correspond to the zones that need to be equipped with sensors. Finally, all the sensor placement results can also be exported in a table structure in the MATLAB workspace.

\subsection{Contaminant Detection and Isolation}

In the Algorithms section, the user can select either the centralized or distributed contaminant detection and isolation algorithms. If the distributed approach is selected, the appropriate interface for configuring the subsystems precedes the Contaminant Detection and Isolation interface shown in Fig. 8. For the presented CDI example, connected subsystems are selected (i.e., at least one airflow path must exist between any two zones inside a subsystem), such that each subsystem represents a group of zones that share physical interconnections and a contaminant can traverse between them. In particular, the building partitioning consisting of the following two subsystems:

$$
\begin{aligned}
& \Sigma_{1}=[Z 1, Z 2, Z 3, Z 4, Z 5], \\
& \Sigma_{2}=[Z 6, Z 7, Z 8, Z 9, Z 10, Z 11, Z 12, Z 13, Z 14] .
\end{aligned}
$$

The two connected subsystems have been selected such that they also have the smallest possible difference in the number of included zones. Thus, the computational complexity is also balanced between the agents of the subsystems. When a building partitioning is selected, the respective indices of zones in the main GUI are colored accordingly. The CDI interface is divided into four sub-sections: i) Uncertainties Bound, ii) Detection and Isolation Parameters, iii) Sensor Noise Bound and iv) Contaminant Detection and Isolation. The Uncertainties Bound sub-section is used for calculating the modeling uncertainties bounds $\left(\overline{\Delta A}_{I}\right.$ and interconnection $\overline{\Delta H}_{I}$ in the case of distributed simulation). These uncertainty bounds can either be given as constant known values, or calculated using random sampling within specific tolerances around the nominal conditions (i.e, wind direction, wind speed, zone temperature and percentage of path openings), or calculated from the scenario file $(* .0)$ as the max deviation from the nominal selection in the generated scenarios (e.g., *.c1,*.c2...). For example, in the interface depicted in Fig. 8, the bounds for the modeling and interconnection uncertainties are calculated by making 100 random selections from within the following parameter ranges: wind speed of $10 \pm 2 \mathrm{~m} / \mathrm{s}$, zone temperatures of $20 \pm 2$ degrees Celsius, wind direction of $90 \pm 10$ degrees from the North, and path openings changing $20 \%$ from the fully open position. The chosen deviations resulted in the following modeling uncertainty bounds: $\overline{\Delta A}_{1}=0.2807, \overline{\Delta A}_{2}=0.0047$ and interconnection uncertainty bounds of $\overline{\Delta H}_{1}=0.2302, \overline{\Delta H}_{2}=0$ for subsystems $\Sigma_{1}$ and $\Sigma_{2}$ respectively. In the Detection and Isolation Parameters sub-section, the user can modify, if required, the default values used by the CDA and CIA illustrated in Fig. 8. These depend on the initial problem assumptions and include the initial maximum state estimation error for detection and isolation, the learning rate and the maximum interval value for estimating the contaminant source, and an initialization value for the estimated source. More information on these parameters can be found in [41]. Finally, a bound on the sensor noise needs to be specified in the Sensor Noise Bound sub-section. Currently, we are assuming uniform, bounded noise, but other types of noise (i.e. Gaussian) can easily be incorporated in the future.

After the uncertainties, CDI parameters and sensor noise bounds are set, the CDI algorithm for all computed contamination scenarios can be executed using the "Run CDI" button appearing on the right bottom corner of Fig. 8. The sequence of calculations performed follows the CDI architecture outlined in Section 2.3. The user is notified for the progress of the detection and isolation algorithms through user friendly graphical displays and message notification banners.

Both centralized and distributed CDI algorithms can be executed for the same simulation files. The results are saved in ".cdi" file types and are annotated with either "_Centralized" or "Distributed" identifiers depending on the algorithm chosen to be executed.

\subsubsection{Contaminant Detection and Isolation Results}

After the execution of the CDI algorithm, the user can view detailed results about the detection and isolation for all the scenarios in the Contaminant Detection and Isolation Results interface as illustrated in Fig. 9. The user can load the results of any specific scenario and view the resulting CDI file. The CDI results interface is comprised of both a table and a list structure. The list includes information for only a specific scenario whereas the table shows results for all the computed scenarios. The user can inspect first the table for identifying a single scenario for further investigation and then select that particular scenario using the "No of Scenarios" drop down menu. For the selected scenario, the detection and isolation time series for specific zones can be plotted. For the case study outlined in Section 4.1 and the scenario parameters given in Section 4.3, the detection results for scenario 17 appear in Fig. 10. The illustrated scenario includes a contaminant source with release rate of 475 $\mathrm{g} / \mathrm{hr}$ which is activated in Z3 from $t=0.95 \mathrm{hr}$ until $t=2.375$ hr. From the results it becomes evident that the contaminant in zone Z3 of the "Holmes house" has been successfully detected since the detection threshold is exceeded. In particular, the contaminant is detected at approximately $t=0.9519$ hours. Following the detection of a contaminant source, the corresponding contaminant isolation agent is activated in order to isolate the contaminant source. As mentioned in Section 2.3, for each zone of a subsystem, a bank of isolators is activated. Since $\Sigma_{1}$ contains five zones as indicated in (11), then a bank of five isolators are in effect. The resulting time series for the isolators in zones Z1 and Z3 appear in Fig. 11a and Fig. 11b respectively.

Each isolator uses the hypothesis that a contaminant source exists in its assigned zone. In order for a contaminant source to be isolated, all but one isolators have to disprove their hypothesis. In Fig. 11a the residual of zone Z3 surpasses the threshold, hence, the assumption of a contaminant source located in $\mathrm{Z} 1$ is 


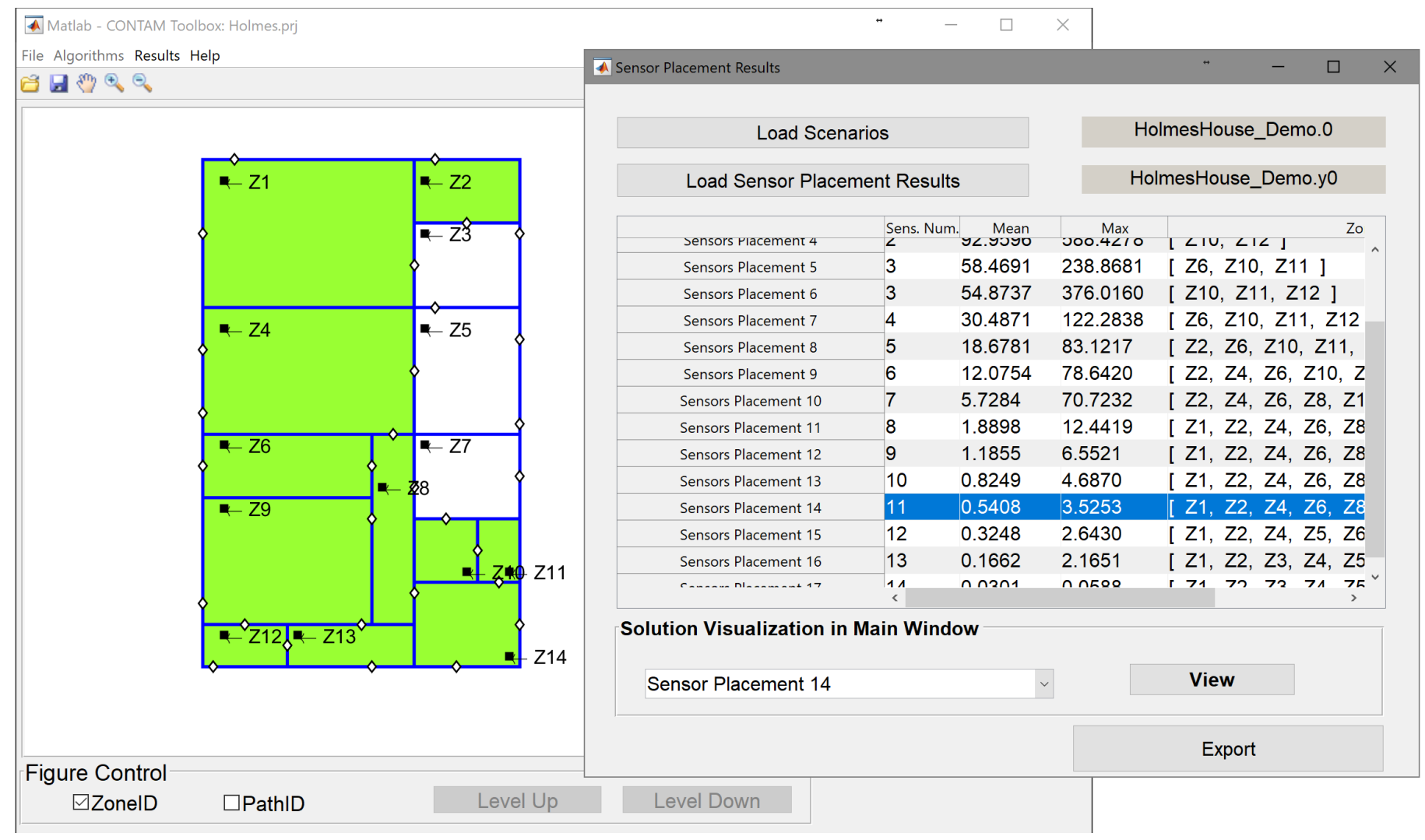

Figure 7: The Sensor Placement solutions interface.

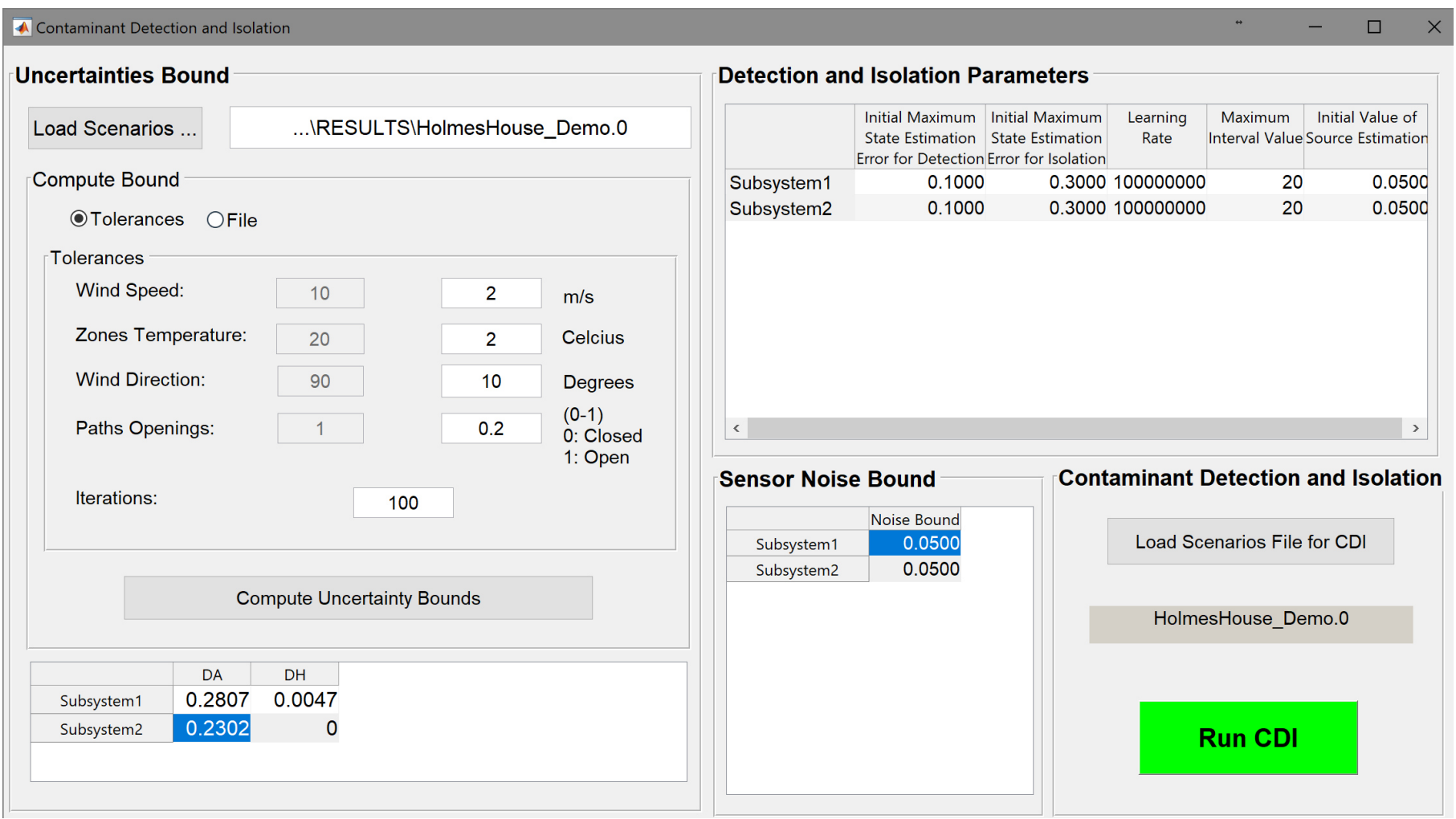

Figure 8: Interface for calculating the uncertainty bounds, setting the CDI parameters and running the CDI algorithm. 


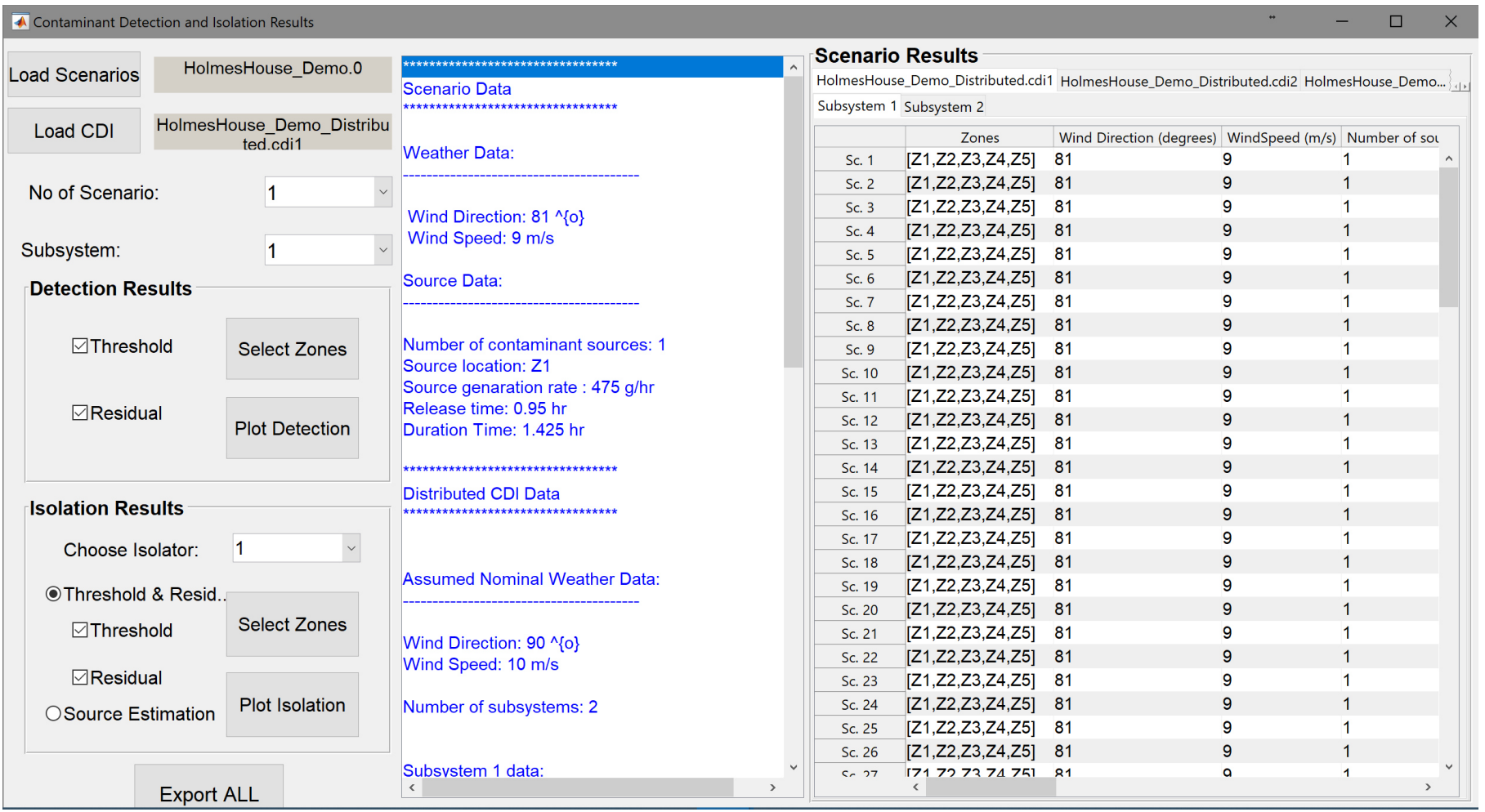

Figure 9: Contaminant Detection and Isolation Results interface for detailed detection and isolation results illustration and export

Z1

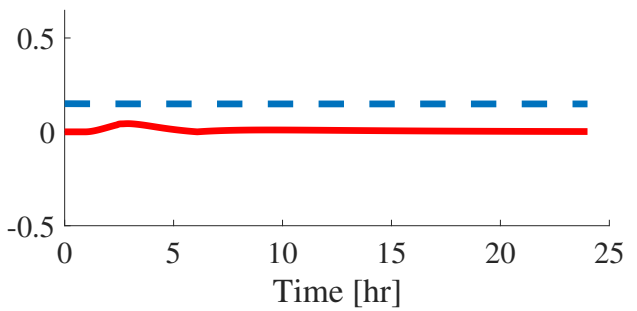

Z3

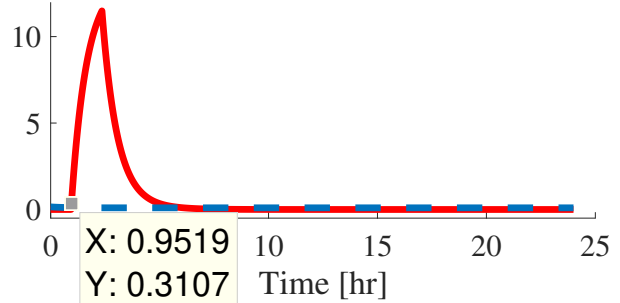

Z5

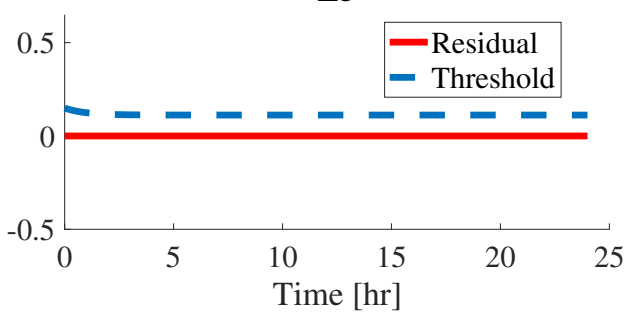

Z2

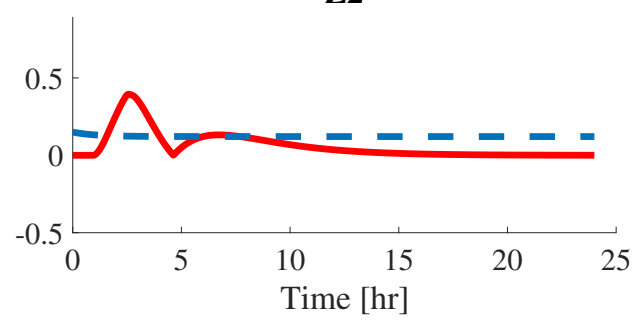

Z4

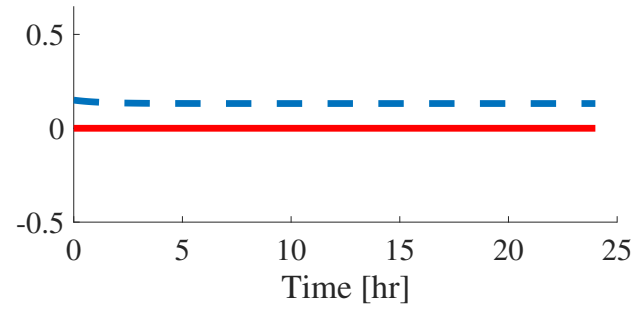

Figure 10: Detection Residuals and Thresholds. The contaminant is detected by Z3. 
Z1

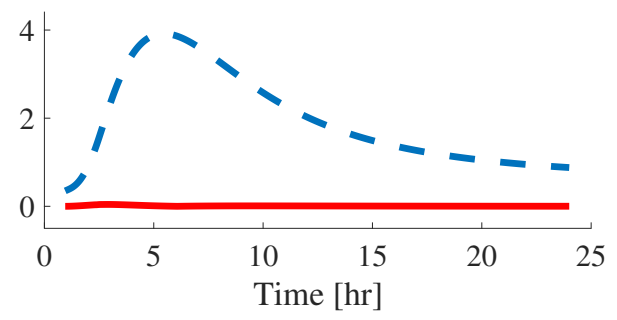

Z3

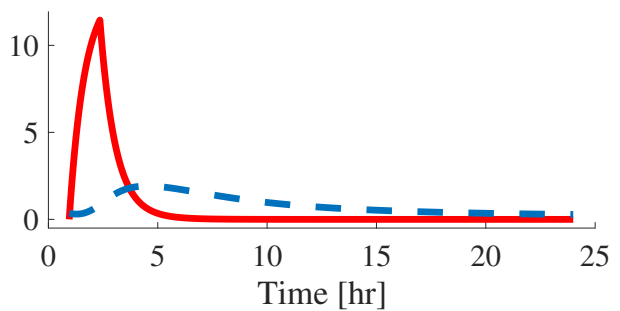

Z5

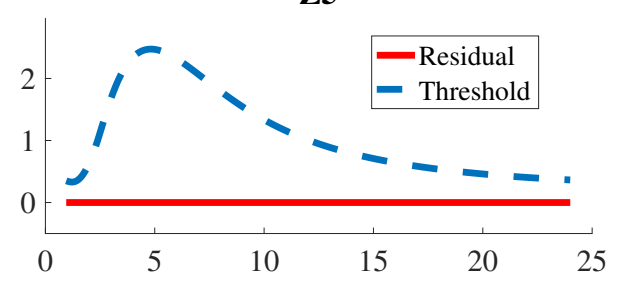

(a)

Z1

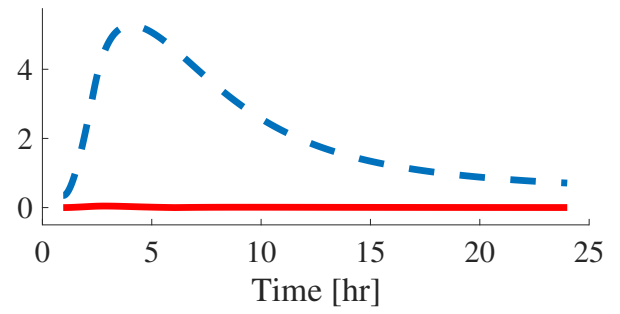

Z3

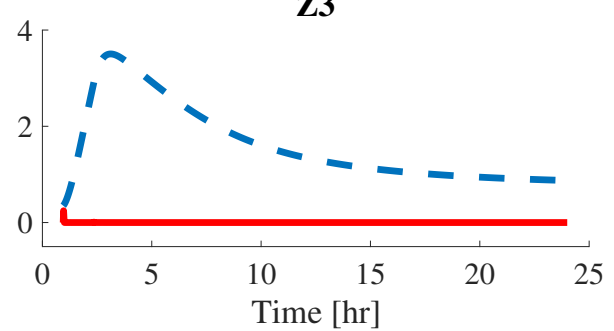

Z5

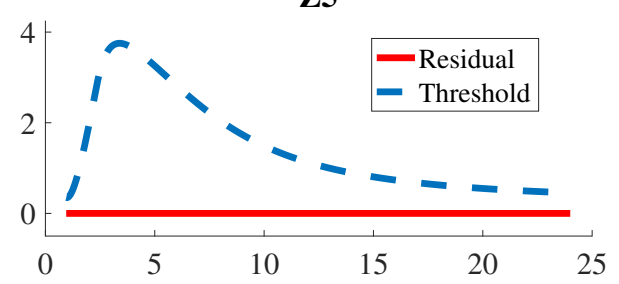

Z2

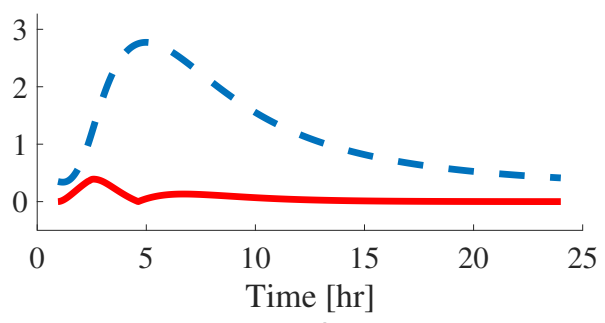

Z4

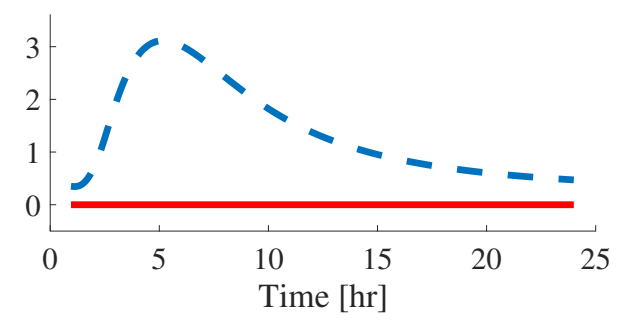

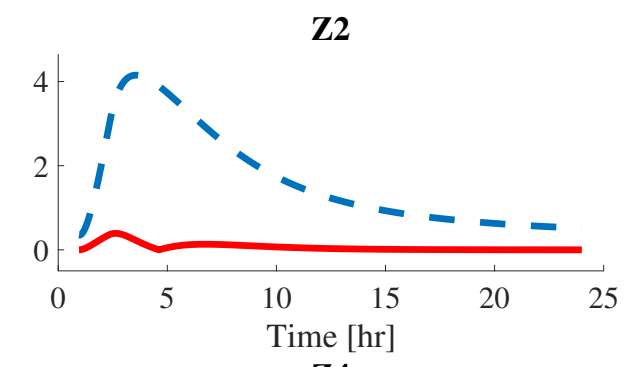

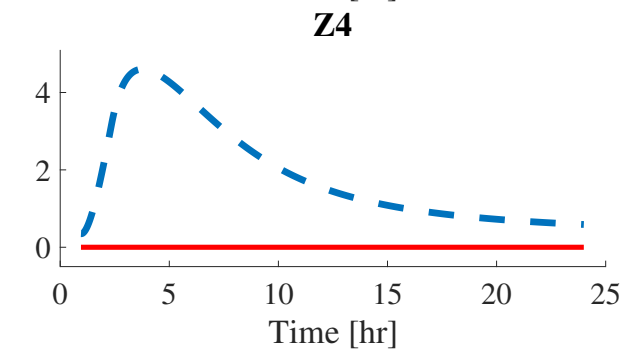

(b)

Figure 11: (a) Bank of Isolators for Z1. Assumption of contaminant existence in Z1 is violated by Z3. (b) Bank of Isolators for Z3. Assumption of contaminant existence in $\mathrm{Z} 3$ is correct. 
wrong. The same response is observed for the isolators of zones Z2, Z4 and Z5. In contrast, the residuals for all zones of the isolator in zone $\mathrm{Z} 3$ remain under the threshold. Therefore the contaminant source is successfully isolated in Z3 at $t=0.99$ hours.

The resulting CDA and CIA figures can be exported through the build-in MATLAB export functions while all the information included in the table as well as the resulting CDA and CIA time series can be exported in a MATLAB table structure.

\section{Conclusions and Future Work}

Contaminant event monitoring in buildings requires large quantities of simulation data to capture the variations in the complex dynamics involved. In this notion, the COMOB toolbox has been developed and released, a software that operates within the MATLAB environment and provides a programming interface for CONTAM, a multi-zone airflow and contaminant transport simulation tool. To facilitate the interaction of the researchers with the COMOB toolbox, an intuitive graphical user interface has been designed for accessing the different functionalities and a modular architecture has been adopted to assist in the implementation of new methods. In this paper, we describe the implementation and evaluation through the toolbox of advanced sensor placement and contaminant detection and isolation algorithms based on the state-space formulation for multi-zone buildings. We demonstrate the toolbox through a case study of a 14-zone real building model referred to as the "Holmes house".

Future directions involve the incorporation of more flexibility in choosing the parameters for constructing the contaminant and airflow scenarios. For the Sensor Placement module, the use of additional impact metrics and response time-delays, as well as different optimization methods for constructing the Pareto Solutions, are among the planned improvements. With respect to the CDI module, recently developed exact and heuristic partitioning algorithms, detailed in [50], used for automatically defining the individual subsystems for the distributed CDI approach, can become valuable future additions. We also plan to incorporate different techniques for detecting and isolating multiple contaminant sources in the same subsystem, as well as differentiating between the presence of contaminant sources and sensor faults. Another interesting future direction, includes the incorporation of CFD capabilities for simulating the indoor environmental conditions. The CFD modeling of the contaminant dispersion in the building interior can provide a detailed and more accurate description of the contaminant dispersion; thus, allowing the fine grained sensor placement and source localization inside each zone. Additionally, one of the aims is to investigate the level of abstraction (i.e., between multi-zone and CFD models) that achieves the right balance between the quality of the CDI solutions and computational complexity.

The ultimate goal of the COMOB toolbox is to serve as a common evaluation framework for related work in contaminant monitoring in buildings. In particular, the simulation of multiple contaminant release scenarios under varying environmental conditions with both multi-zone and CFD models and the extraction of the results into well defined data structures can serve as benchmarks for evaluating the performance of the various developed algorithms.

\section{Acknowledgments}

This work has been supported by the European Research Council (ERC) under the European Unions Horizon 2020 research and innovation programme grant agreement No 755134 (Domognostics), by the European Unions Horizon 2020 research and innovation programme under grant agreement No 739551 (KIOS CoE) and by the Republic of Cyprus through the Directorate General for European Programmes, Coordination and Development.

\section{Appendix A. Supplementary material}

A tutorial video that illustrates step-by-step the process of using the COMOB toolbox and demonstrates its different functionalities.

\section{Software availability}

Name of software: COMOB (COntaminat MOnitoring in Buildings)

Version: 2.0 - tested on MATLAB R2018a

Developers: Marinos Christoloulou, Marios Kyriakou, and Alexis Kyriacou

Contact email: akyria09@ucy.ac.cy (Alexis Kyriacou)

Year first available: 2013

Availability: Free of charge and can be downloaded from the GitHub repository

https : //github. com/KIOS-Research/COMOB-Toolbox Hardware Requirements: Any computer with at least 2 logical processing cores and 4GB of RAM

Software Requirements: MATLAB, CONTAM

Dependencies: The developed software has the following MATLAB toolbox dependencies; a) Control Systems, b) Curve Fitting, c) Global Optimization, d) Parallel Computing, e) Statistics and Machine Learning, and f) Symbolic Math. 
[1] N. E. Klepeis, W. C. Nelson, W. R. Ott, J. P. Robinson, A. M. Tsang, P. Switzer, J. V. Behar, S. C. Hern, W. H. Engelmann, The National Human Activity Pattern Survey (NHAPS): a resource for assessing exposure to environmental pollutants, Journal of Exposure Analysis and Environmental Epidemiology 11 (3) (2001) 231-252. doi:10.1038/s j . jea. 7500165.

[2] Z. Ren, J. Stewart, Prediction of personal exposure to contaminant sources in industrial buildings using a sub-zonal model, Environmental Modelling \& Software 20 (5) (2005) 623-638. doi:10.1016/j.envsoft. 2004. 03.007.

[3] H. E. Feustel, COMIS - an international multizone air-flow and contaminant transport model, Energy and Buildings 30 (1) (1999) 3-18. doi:10.1016/S0378-7788(98)00043-7.

[4] W. S. Dols, B. J. Polidoro, CONTAM user guide and program documentation version 3.2, NIST Technical Note 1887, US National Institude of Standards and Technology (Sep. 2015). doi : 10.6028/NIST. TN . 1887.

[5] B. Chakrabarty, Computer-aided design in urban development and management - A software for integrated planning and design by optimization, Building and Environment 42 (1) (2007) 473-494. doi:10.1016/j. buildenv.2005.08.010.

[6] O. Gutschker, Parameter identification with the software package LORD, Building and Environment 43 (2) (2008) 163-169. doi:10.1016/j . buildenv. 2006.10.010.

[7] M. J. Jiménez, H. Madsen, K. K. Andersen, Identification of the main thermal characteristics of building components using MATLAB, Building and Environment 43 (2) (2008) 170-180. doi:10.1016/j . buildenv . 2006.10.030.

[8] X. Pang, M. Wetter, P. Bhattacharya, P. Haves, A framework for simulation-based real-time whole building performance assessment, Building and Environment 54 (2012) 100-108. doi:10.1016/j. buildenv. 2012.02.003.

[9] D. Calì, M. T. Wesseling, D. Müller, WinProGen: A Markov-Chain-based stochastic window status profile generator for the simulation of realistic energy performance in buildings, Building and Environment 136 (2018) 240-258. doi:10.1016/j.buildenv.2018.03.048.

[10] R. Kumanayake, H. Luo, A tool for assessing life cycle $\mathrm{CO}_{2}$ emissions of buildings in Sri Lanka, Building and Environment 128 (2018) 272-286. doi:10.1016/j.buildenv.2017.11.042.

[11] A. N. Gade, T. S. Larsen, S. B. Nissen, R. L. Jensen, REDIS: A valuebased decision support tool for renovation of building portfolios, Building and Environment 142 (2018) 107-118. doi:10.1016/j.buildenv . 2018.06 .016

[12] Y. L. Chen, J. Wen, Sensor system design for building indoor air protection, Building and Environment 43 (7) (2008) 1278-1285. doi: 10.1016/j.buildenv.2007.03.011.

[13] X. Liu, Z. J. Zhai, Protecting a whole building from critical indoor contamination with optimal sensor network design and source identification methods, Building and Environment 44 (11) (2009) 2276-2283. doi:10.1016/j.buildenv.2009.03.009.

[14] Y. L. Chen, J. Wen, Comparison of sensor systems designed using multizone, zonal, and CFD data for protection of indoor environments, Building and Environment 45 (4) (2010) 1061-1071. doi:10.1016/j. buildenv. 2009.10.015.

[15] Y. L. Chen, J. Wen, The selection of the most appropriate airflow model for designing indoor air sensor systems, Building and Environment 50 (2012) 34-43. doi:10.1016/j. buildenv. 2011.10.007.

[16] D. Eliades, M. Michaelides, C. Panayiotou, M. Polycarpou, Securityoriented sensor placement in intelligent buildings, Building and Environment 63 (2013) 114-121. doi : 10.1016/j . buildenv . 2013.02 .006.

[17] A. D. Fontanini, U. Vaidya, B. Ganapathysubramanian, A methodology for optimal placement of sensors in enclosed environments: A dynamical systems approach, Building and Environment 100 (2016) 145-161. doi : 10.1016/j. buildenv. 2016.02.003.

[18] J. Waeytens, S. Sadr, Computer-aided placement of air quality sensors using adjoint framework and sensor features to localize indoor source emission, Building and Environment 144 (2018) 184-193. doi:10. 1016/j.buildenv. 2018.08.012.

[19] M. Awadalla, T.-F. Lu, Z. F. Tian, B. Dally, Z. Liu, 3D framework combining CFD and MATLAB techniques for plume source localization research, Building and Environment 70 (2013) 10-19. doi:10.1016/j . buildenv.2013.07.021.
[20] Y. Chen, H. Cai, Z. Chen, Q. Feng, Using multi-robot active olfaction method to locate time-varying contaminant source in indoor environment, Building and Environment 118 (2017) 101-112. doi:10.1016/ j.buildenv.2017.03.030.

[21] Q. Feng, H. Cai, F. Li, X. Liu, S. Liu, J. Xu, An improved particle swarm optimization method for locating time-varying indoor particle sources, Building and Environment 147 (2019) 146-157. doi: 10.1016/j.buildenv. 2018.10.008

[22] V. Vukovic, J. Srebric, Application of neural networks trained with multizone models for fast detection of contaminant source position in buildings, ASHRAE Transactions 113 (2007) 154-162.

[23] V. Vukovic, P. C. Tabares-Velasco, J. Srebric, Real-time identification of indoor pollutant source positions based on neural network locator of contaminant sources and optimized sensor networks, Journal of the Air \& Waste Management Association 60 (9) (2010) 1034-1048. doi : 10.3155/1047-3289.60.9.1034

[24] H. Cai, X. Li, Z. Chen, M. Wang, Rapid identification of multiple constantly-released contaminant sources in indoor environments with unknown release time, Building and Environment 81 (2014) 7-19. doi : 10.1016/j.buildenv.2014.06.006.

[25] M. P. Michaelides, V. Reppa, M. Christodoulou, C. G. Panayiotou, M. M. Polycarpou, Contaminant event monitoring in multi-zone buildings using the state-space method, Building and Environment 71 (2014) 140-152. doi:doi.org/10.1016/j.buildenv.2013.09.019.

[26] A. Kyriacou, M. P. Michaelides, V. Reppa, S. Timotheou, C. G. Panayiotou, M. M. Polycarpou, Distributed contaminant detection and isolation for intelligent buildings, IEEE Transactions on Control Systems Technology 26 (6) (2018) 1925-1941. doi : 10.1109/TCST . 2017 . 2754986.

[27] Z. Z. X. Liu, Inverse modeling methods for indoor airborne pollutant tracking: literature review and fundamentals, Indoor Air 17 (6) (2007) 419-438. arXiv:https://onlinelibrary.wiley.com/ doi/pdf/10.1111/j.1600-0668.2007.00497.x, doi:10.1111/j. 1600-0668.2007.00497.x.

[28] T. T. Zhang, H. Li, S. Wang, Inversely tracking indoor airborne particles to locate their release sources, Atmospheric environment 55 (2012) 328338. doi:10.1016/j. atmosenv.2012.03.066.

[29] H. Wang, S. Lu, J. Cheng, Z. J. Zhai, Inverse modeling of indoor instantaneous airborne contaminant source location with adjoint probabilitybased method under dynamic airflow field, Building and Environment 117 (2017) 178-190. doi:10.1016/j.buildenv.2017.03.017.

[30] Y. Wei, H. Zhou, T. T. Zhang, S. Wang, Inverse identification of multiple temporal sources releasing the same tracer gaseous pollutant, Building and Environment 118 (2017) 184-195. doi:10.1016/j.buildenv. 2017.03 .026$.

[31] L. Zeng, J. Gao, B. Du, R. Zhang, X. Zhang, Probability-based inverse characterization of the instantaneous pollutant source within a ventilation system, Building and Environment 143 (2018) 378-389. doi :10 .1016/ j.buildenv.2018.07.036.

[32] T. Matsuo, A. Kondo, H. Shimadera, T. Kyuno, Y. Inoue, Estimation of indoor contamination source location by using variational continuous assimilation method, Building Simulation 8 (4) (2015) 443-452. doi : 10.1007/s12273-015-0221-z.

[33] T. Matsuo, H. Shimadera, A. Kondo, Identification of multiple contamination sources using variational continuous assimilation, Building and Environment 147 (2019) 422-433. doi:10.1016/j.buildenv.2018.10. 041.

[34] Y. Park, P. M. Tagade, H. Choi, Deep gaussian process-based bayesian inference for contaminant source localization, IEEE Access 6 (2018) 49432-49449. doi:10.1109/ACCESS . 2018. 2867687.

[35] K. Fong, V. Hanby, T. Chow, HVAC system optimization for energy management by evolutionary programming, Energy and Buildings 38 (3) (2006) 220-231. doi:10.1016/j . enbuild.2005.05.008.

[36] A. Dounis, C. Caraiscos, Advanced control systems engineering for energy and comfort management in a building environment - A review, Renewable and Sustainable Energy Reviews 13 (6-7) (2009) 1246-1261. doi: 10.1016/j.rser.2008.09.015.

[37] S. Wong, K. K. Wan, T. N. Lam, Artificial neural networks for energy analysis of office buildings with daylighting, Applied Energy 87 (2) (2010) 551-557. doi:10.1016/j.apenergy.2009.06.028.

[38] G. Boracchi, M. Michaelides, M. Roveri, A cognitive monitoring system for detecting and isolating contaminants and faults in intelligent buildings, 
IEEE Transactions on Systems, Man, and Cybernetics: Systems 48 (3) (2018) 433-447. doi : 10.1109/TSMC . 2016.2608419.

[39] L. Wang, W. Dols, Q. Chen, Using CFD capabilities of CONTAM 3.0 for simulating airflow and contaminant transport in and around buildings, HVAC\&R Research 16 (6) (2010) 749-763.

[40] M. Michaelides, V. Reppa, C. Panayiotou, M. Polycarpou, Contaminant event monitoring in intelligent buildings using a multi-zone formulation, in: Proceedings of the 8th IFAC Symposium on Fault Detection, Supervision and Safety of Technical Processes (SAFEPROCESS), Vol. 45, Mexico City, Mexico, 2012, pp. 492-497. doi:10. 3182/20120829-3-MX-2028.00100.

[41] X. Zhang, M. M. Polycarpou, T. Parisini, Design and analysis of a fault isolation scheme for a class of uncertain nonlinear systems, Annual Reviews in Control 32 (1) (2008) 107-121. doi:10.1016/j . arcontrol. 2008.03 .007$.

[42] U.S. Environmental Protection Agency (EPA), Exposure factors handbook: 2011 edition, National Center for Environmental Assessment, Washington, DC, EPA/600/R-09/052F (Sep. 2011).

[43] V. Venkatasubramanian, R. Rengaswamy, K. Yin, S. N. Kavuri, A review of process fault detection and diagnosis: Part I: Quantitative model-based methods, Computers \& Chemical Engineering 27 (3) (2003) 293-311. doi: 10.1016/S0098-1354(02)00160-6.

[44] V. Venkatasubramanian, R. Rengaswamy, S. N. Kavuri, A review of process fault detection and diagnosis: Part II: Qualitative models and search strategies, Computers \& Chemical Engineering 27 (3) (2003) 313-326. doi:10.1016/S0098-1354(02)00161-8.

[45] I. Hwang, S. Kim, Y. Kim, C. E. Seah, A survey of fault detection, isolation, and reconfiguration methods, IEEE Transactions on Control Systems Technology 18 (3) (2010) 636-653. doi:10.1109/TCST.2009. 2026285.

[46] Z. Gao, C. Cecati, S. X. Ding, A survey of fault diagnosis and faulttolerant techniques-Part I: fault diagnosis with model-based and signalbased approaches, IEEE Transactions on Industrial Electronics 62 (6) (2015) 3757-3767. doi:10.1109/TIE. 2015. 2417501.

[47] M. Blanke, M. Kinnaert, J. Lunze, M. Staroswiecki, Diagnosis and FaultTolerant Control, 2nd Edition, Springer-Verlag Berlin Heidelberg, 2006. doi : 10.1007/978-3-540-35653-0.

[48] M. P. Michaelides, D. G. Eliades, M. Christodoulou, M. Kyriakou, C. Panayiotou, M. Polycarpou, A MATLAB-CONTAM Toolbox for Contaminant Event Monitoring in Intelligent Buildings, in: Proceedings of the 9th IFIP WG 12.5 International Conference on Artificial Intelligence Applications and Innovations, Paphos, Cyprus, 2013, pp. 605-614. doi: 10. 1007/978-3-642-41142-7_61.

[49] D. G. Eliades, M. P. Michaelides, M. Christodoulou, M. Kyriakou, C. G. Panayiotou, M. M. Polycarpou, An indoor contaminant sensor placement toolbox for critical infrastructure buildings, in: Proceedings of the 8th International Workshop on Critical Information Infrastructures Security (CRITIS), Amsterdam, The Netherlands, 2013, pp. 179-190. doi:10. 1007/978-3-319-03964-0_16.

[50] A. Kyriacou, S. Timotheou, M. P. Michaelides, C. Panayiotou, M. Polycarpou, Partitioning of intelligent buildings for distributed contaminant detection and isolation, IEEE Transactions on Emerging Topics in Computational Intelligence 1 (2) (2017) 72-86. doi : 10 . 1109/TETCI . 2017. 2665119. 